\title{
A Modified Learning Algorithm Incorporating Additional Functional Constraints Into Neural Networks
}

\author{
Fei Han ${ }^{1,2}$ De-Shuang Huang ${ }^{1}$ Xu-Qing Li ${ }^{1}$ Michael R. Lyu ${ }^{3}$ Tat-Ming Lok ${ }^{4}$ \\ 1. Hefei Institute of Intelligent Machines, Chinese Academy of Sciences, P.O.Box 1130, \\ Hefei Anhui 230031, China \\ hanfei1976@iim.ac.cn \\ 2. Department of Automation, University of Science and Technology of China, Hefei \\ 230027, China \\ 3.Computer Science \& Engineering Dept., The Chinese University of Hong Kong, Shatin, \\ Hong Kong \\ 4.Information Engineering Dept., The Chinese University of Hong Kong, Shatin, Hong \\ Kong
}

\begin{abstract}
In this paper, a modified learning algorithm to obtain better generalization performance is proposed. The cost terms of this new algorithm are selected based on the second-order derivatives of the neural activation at the hidden layers and the first-order derivatives of the neural activation at the output layer. It can be guaranteed that in the course of training, the additional cost terms for this algorithm can penalize both the input-to-output mapping sensitivity and the high frequency components to obtain better generalization performance. Finally, theoretical justifications and simulation results are given to verify the efficiency and effectiveness of the proposed learning algorithm.
\end{abstract}

\section{Introduction}

Most traditional learning algorithms with feedforward neural networks (FNN) are to use the sum-of-square error criterion to derive the updated formulae. However, these learning algorithms have not considered the network structure and the involved problem properties, thus their capabilities are limited [1]. In order to obtain better generalization capability [2-3], many constrained learning algorithms that incorporate additional functional constraints into neural networks have been proposed in literature [4-9].

In literature [10], two learning algorithms were proposed that are referred to as Hybrid-I method and Hybrid-II method, respectively. The Hybrid-I algorithm incorporates the first-order derivatives of the neural activation at hidden layers into the sum-of-square error cost function to reduce the input-to-output mapping sensitivity. On the other hand, the Hybrid-II algorithm incorporates the second-order derivatives of the neural activation at hidden layers and output layer into the sum-ofsquare error cost function to penalize the high frequency components in training data. Nevertheless, all the above learning algorithms can almost improve the generalization performance to some degree, but not having the best generalization performance. 
In this paper, a new modified learning algorithm based on Hybrid-I and Hybrid-II algorithms is proposed. The additional cost terms of the new algorithm are selected based on the first-order derivatives of the neural activation at the output layer and the second-order derivatives of the neural activation at the hidden layers. This new algorithm inherits the features from the original Hybrid-I and Hybrid-II algorithms. Moreover, through experiments, it can be found that the generalization performance for this modified algorithm is better than the one for the original Hybrid ones.

\section{The New Modified Learning Algorithm}

Considering an FNN with one input layer, $L-1$ hidden layers, and one output layer, the units in each layer apart from the input layer receive the inputs from all units in the previous layer. For simplicity, the same activation function for all neurons at all layers, i.e., tangent sigmoid transfer function is adopted:

$$
f(x)=(1-\exp (-2 x)) /(1+\exp (-2 x)) \text {. }
$$

It can be deduced that this activation function has the following property:

$$
f^{\prime \prime}(x)=-2 f(x) f^{\prime}(x) .
$$

Before presenting input-to-output sensitivity, the following mathematical notation is made first. Assume that $x_{k}$ and $y_{i}$ denote the $k$ th element of the input vector and the $i$ th element of the output vector, respectively; $w_{j_{l} j_{l-1}}$ denotes the synaptic weight from the $j_{l}$ th hidden neuron at the $l$ th layer to the $j_{l-1}$ th hidden neuron at $(l-1)$ th layer; $w_{i j_{L-1}}$ denotes the synaptic weight from the $i$ th neuron at output layer to the $j_{L-1}$ th hidden neuron at $(L-1)$ th layer; $w_{j_{1} k}$ denotes the synaptic weight from the $j_{l}$ th hidden neuron at the first layer to the $k$ th element of the input vector; $f_{l}^{\prime}(\cdot)$ is the derivative of the sigmoid function $f_{l}(\cdot)$ at $l$ th layer; $h_{j_{l}}=f_{l}\left(\hat{h}_{j_{l}}\right)$ is the activation function of the $j_{l}$ th element at the $l$ th layer with $\hat{h}_{j_{l}}=\sum_{j_{t-1}} w_{j_{l} j_{t-1}} h_{j_{t-1}}$. The $t_{i}$ and $y_{i}$ denote the target and actual output values of the $i$ th neuron at output layer, respectively; $N_{l}$ denotes the number of the neurons at the $l$ th layer.

To obtain better generalization performance than Hybrid-I and Hybrid-II algorithms, a new cost function containing the additional output layer penalty term and the weights decay term at the hidden layers is defined as follow:

$$
E=\frac{1}{N} \sum_{S=1}^{N} E^{s},
$$

where

$$
E^{S}=\frac{1}{2 N_{L}} \sum_{i}\left(t_{i}^{S}-y_{i}^{S}\right)^{2}+\sum_{l=1}^{L-1} \gamma_{l} E_{h}^{I S}+\frac{\gamma_{L}}{N_{L}} \sum_{j_{L}}^{N_{L}} f^{\prime}\left(\hat{h}_{j_{l}}^{I S}\right),
$$

and 
A Modified Learning Algorithm Incorporating Additional Functional Constraints Into Neural Networks 3

$$
E_{h}^{I S}=\frac{1}{N_{l}} \sum_{j_{l}}^{N_{l}} f^{\prime}\left(\hat{h}_{j_{l}}^{I S}\right)\left[\frac{1}{2} \sum_{j_{l-1}=1}^{N_{L-1}}\left(w_{j_{l} j_{l-1}}^{S}\right)^{2}\right]
$$

The cost function $E^{S}$ denotes the corresponding cost function for the $S$ th stored pattern. The second term in right side of Eqn. (4) is a kind of weights decay term; the third term in right side of Eqn. (4) denotes the additional output layer penalty term at the output layer; the gains $\gamma_{1}$ and $\gamma_{L}$ represent the relative significance among the cost terms; $\mathrm{N}$ denotes the number of the stored patterns.

The network is trained by a steepest-descent error minimization algorithm, the synaptic weight update for $s$ th stored pattern becomes into:

$$
\Delta w_{j_{l} j_{l-1}}^{S}=-\eta_{l} \frac{\partial E^{S}}{\partial w_{j_{l} j_{l-1}}^{S}}=\eta_{l} \delta_{j_{l}}^{S} h_{j_{l-1}}^{S}-\eta_{l} \frac{\gamma_{l}}{N_{l}} w_{j_{l} j_{l-1}}^{S} f^{\prime}\left(\hat{h}_{j_{l-1}}^{S}\right),
$$

where $\delta_{j_{1}}^{s}$ denotes the negative derivative of the cost $E^{s}$ to the $\hat{h}_{j_{1}}^{s}$ at $l$ th layer.

The negative derivative of the cost $E^{s}$ to the $\hat{h}_{j_{1}}^{s}$ at the hidden layer, i,e., $\delta_{j_{1}}^{s}$, can be computed by back-propagation style as follows:

$$
\delta_{j_{l}}^{S}=-\frac{\partial E}{\partial \hat{h}_{j_{l}}^{S}}=\sum_{j_{l+1}=1}^{N_{l+1}} \delta_{j_{l+1}}^{S} w_{j_{l+1} j_{l}}^{S} f^{\prime}\left(\hat{h}_{j_{l}}^{S}\right)-\frac{\gamma_{l}}{N_{l}} f^{\prime \prime}\left(\hat{h}_{j_{l}}^{S}\right) \frac{1}{2} \sum_{j_{j_{l-1}=1}=1}^{N_{L-1}}\left(w_{j_{l} j_{l-1}}^{S}\right)^{2}, l=1, \cdots, L-1 .
$$

The negative derivative of the cost $E^{S}$ to the $\hat{h}_{j_{l}}^{S}$ at the output layer, i.e., $\delta_{j_{L}}^{S}$, can be calculated as follows:

$$
\delta_{j_{L}}^{S}=-\frac{\partial E^{S}}{\partial \hat{h}_{j_{L}}^{S}}=\frac{1}{N_{L}} f^{\prime}\left(\hat{h}_{j_{L}}^{S}\right)\left(t_{j_{L}}^{S}-y_{j_{L}}^{S}\right)-\frac{\gamma_{L}}{N_{L}} f^{\prime \prime}\left(\hat{h}_{j_{L}}^{S}\right) .
$$

\section{Theoretical Analysis for This Modified Learning Algorithm}

According to literature [11], for an $L$-layered feedforward neural network, the sensitivity for $y_{i}$ to $x_{k}$ can be defined as:

$$
\frac{\partial y_{i}}{\partial x_{k}}=\sum_{j_{1}, \cdots, j_{L-1}} w_{i j_{L-1}} w_{j_{L-1} j_{L-2}} \cdots w_{j_{1} k} f_{L}^{\prime}\left(\hat{y}_{i}\right) f_{L-1}^{\prime}\left(\hat{h}_{j_{L-1}}\right) \cdots f_{1}^{\prime}\left(\hat{h}_{j_{1}}\right) \cdot
$$

From this equation, it can be deduced that while $\hat{h}_{j_{l}}$ becomes bigger, the derivative $f_{l}^{\prime}\left(\hat{h}_{j_{l}}\right)$ may become smaller sharply. As a result, the low input-to-output sensitivity will be achieved. For simplicity, consider a single-layered neural network with tangent sigmoid neuron. If the input vector $x$ is modified by $\Delta x$, the change $\Delta y_{i}$, at the ith output neuron may be approximated as: 


$$
\Delta y_{i}=y_{i}(x+\Delta x)-y_{i}(x) \approx \sum_{k} \Delta X_{k} \frac{\partial y_{i}}{\partial X_{k}}=\sum_{k} w_{i k} f^{\prime}\left(\hat{y}_{i}\right) \Delta_{X_{k}} .
$$

Consequently, $\Delta y_{i} / y_{i}$ can be computed as follow:

$$
\frac{\Delta y_{i}}{y_{i}}=\frac{\sum_{k} w_{i k} f^{\prime}\left(\hat{y}_{i}\right) \Delta X_{k}}{f\left(\hat{y}_{i}\right)}=\frac{f^{\prime}\left(\hat{y}_{i}\right)}{f\left(\hat{y}_{i}\right)} \sum_{k} w_{i k} X_{k} \frac{\Delta X_{k}}{X_{k}}=\frac{f^{\prime}\left(\hat{y}_{i}\right) \hat{y}_{i}}{f\left(\hat{y}_{i}\right)} \frac{\Delta X_{k}}{x_{k}} .
$$

$g\left(\hat{y}_{i}\right)$ is defined as:

$$
g\left(\hat{y}_{i}\right)=\frac{f^{\prime}\left(\hat{y}_{i}\right) \hat{y}_{i}}{f\left(\hat{y}_{i}\right)}=\frac{4 \hat{y}_{i}}{\exp \left(2 \hat{y}_{i}\right)-\exp \left(-2 \hat{y}_{i}\right)} .
$$

The $g\left(\hat{y}_{i}\right)$ has generally a maximum at $\hat{y}_{i}=0$ and two minima at $\hat{y}_{i}= \pm \infty$. When the value of $\hat{y}_{i}$ becomes larger, the value of $g\left(\hat{y}_{i}\right)$ becomes exponentially decreasing. Consequently, a larger value of $\hat{y}_{i}$ brings on better generalization capability. Apparently, it can be seen that the $g\left(\hat{y}_{i}\right)$ and $f^{\prime}\left(\hat{y}_{i}\right)$ have similar functional forms according to literature [10], thus, the second additional cost term in Eqn. (4) can result in better generalization performance. As far as an $L$-layered feedforward neural network is concerned, the same result can be obtained. According to the above results, the network obtains lower input-to-output sensitivity in the first hidden layer, and it means that the changes of the input vector will lead to smaller changes of the values of output vector in the first hidden layer. In the similar way, the smaller changes in the first hidden layer will result in much smaller changes of the values of the output vector in the second hidden layer, because the output vector in the first hidden layer is the input vector for the second hidden layer. The remaining layers may be deduced by analogy. Hence, it can be easily seen that the values of the output vector in the output layer get much smaller changes although the input vector is changed a lot.

From a Bayesian perspective, all the additional cost functions designed for the above constrained learning algorithms can be interpreted as a negative logarithm of the prior probability distribution of weights [12-13]. In order to obtain good generalization capability, this new hybrid algorithm tries to reduce the network complexity by introducing weight decay term. For the weight decay form, $E_{c}(w)=\frac{1}{2} \sum_{i} w_{i}^{2}$, it can be derived by taking negative logarithm on the Gaussian distribution of weights. This weight decay method penalizes large weights and rewards small weights, but it decays weights at the same rates regardless of its sizes [10]. For simplicity, the weight decay terms in the new hybrid learning algorithm, i.e., the first additional cost term in Eqn. (4), can be simplified as: $E_{c}(w)=\frac{f^{\prime}}{2} \sum_{i} w_{i}^{2}$. It favors large weights only when the corresponding hidden activation is saturated. The derivative of hidden activation, $f^{\prime}$, can be regarded as a scaling parameter to control whether weights are scaled up or down during learning process .

According to the above results, it can be concluded that in the course of training, the proposed learning algorithm can obtain better generalization performance by 
A Modified Learning Algorithm Incorporating Additional Functional Constraints Into Neural

Networks 5

penalizing both the input-to-output mapping sensitivity and high frequency components in training data.

\section{Experimental Results and Discussion}

To demonstrate the improved generalization capability of the proposed modified learning algorithm, in the following, experiments with two real-world benchmarks of sunspot time series and chaotic laser pulsation data will be done. The latter is obtained from Santa Fe competition data set A.

\subsection{Single-step and Iterative-step Prediction for Sunspot Time Series}

To compare the generalization ability of the proposed learning algorithm with the one of the two original Hybrid ones, a (12-8-1)-sized network to solve the sunspot time series single-step and iterative-step prediction is used. Assume that sunspot data from the year 1700 to1920 are used as training set. The data after the year 1920 are used as testing set. In addition, as for single-step prediction, this testing data is divided into four intervals, that is, from the year 1921 to 1955, 1956 to1979, 1980 to 2003, and finally 1921 to 2003. As a result, the single-step prediction results are shown in Figs. 1-3 for Hybrid-I algorithm, Hybrid-II algorithm and the proposed new learning algorithm, respectively. In the meantime, the iterative prediction results are shown in Figs. 4-6 for above three learning algorithms.

In order to statistically compare the prediction accuracies for sunspot data with the four algorithms (listed in Table1 and Table 2), experiment is done fifty times for each algorithm and then calculates its average accuracy value. The corresponding results are summarized in Table 1 and Table 2 for single-step prediction and iterative-step prediction. From these results, it can be seen apparently that the proposed learning algorithm has better generalization capability than the BP algorithm as well as the two original hybrid algorithms, because the mean squared errors of the modified algorithm for the testing data set is smaller than the ones for the other learning ones.

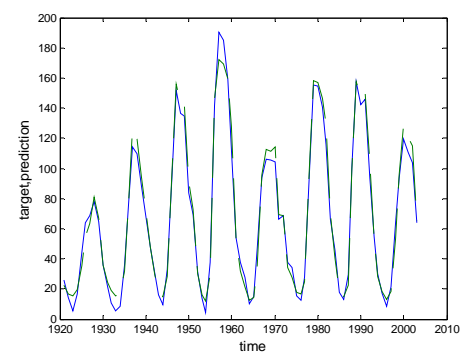

(a) The predicted values

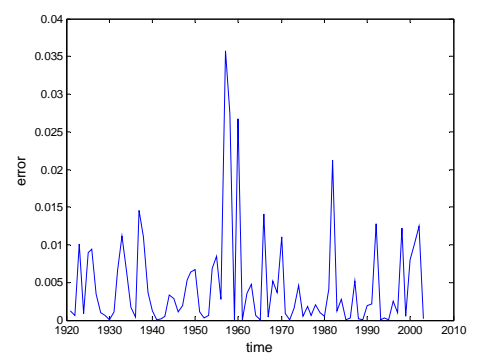

(b) The predicted errors

Fig. 1. Results with single-step prediction for sunspot time series by using Hybrid-I algorithm 
Fei Han1, 2 De-Shuang Huang 1 Xu-Qing Li 1 Michael R. Lyu3 Tat-Ming Lok 4

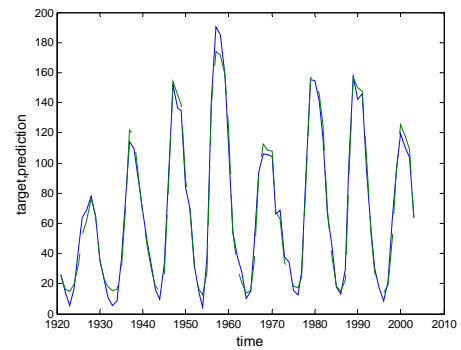

(a) The predicted values

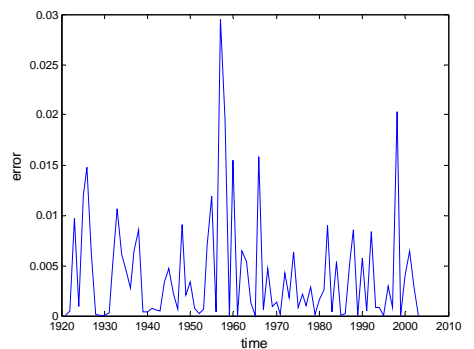

(b) The predicted errors

Fig. 2. Results with single-step prediction for sunspot time series by using Hybrid-II algorithm

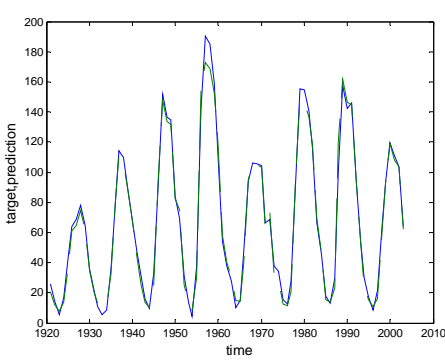

(a) The predicted values

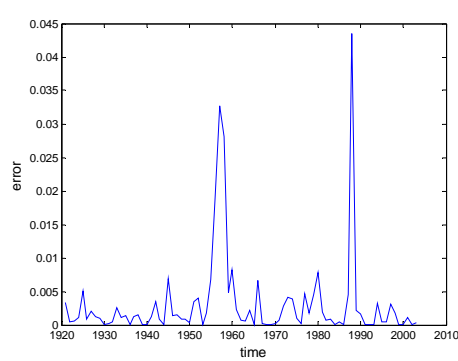

(b) The predicted errors

Fig. 3. Results with single-step prediction for sunspot time series by using the new learning algorithm

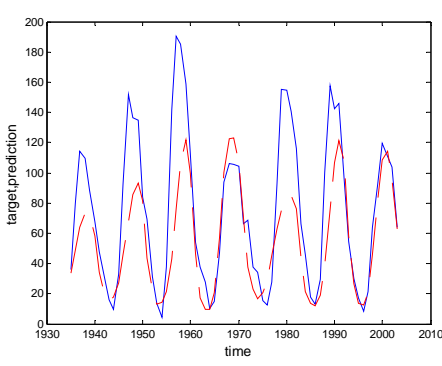

(a) The predicted values

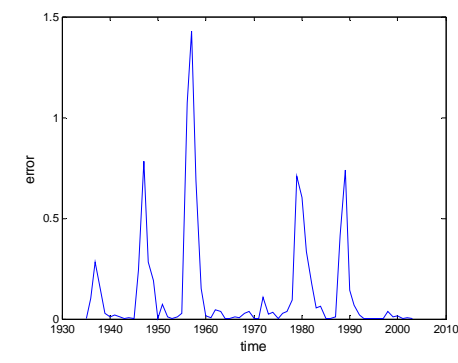

(b) The predicted errors

Fig. 4. Results with iterative-step prediction for sunspot time series by using Hybrid-I algorithm 
A Modified Learning Algorithm Incorporating Additional Functional Constraints Into Neural Networks 7

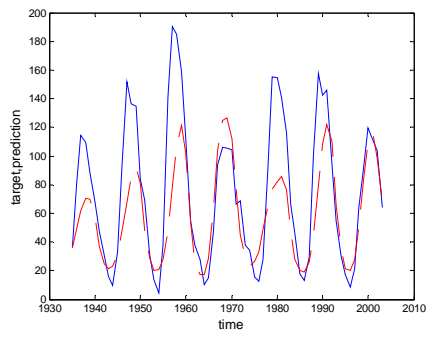

(a) The predicted values

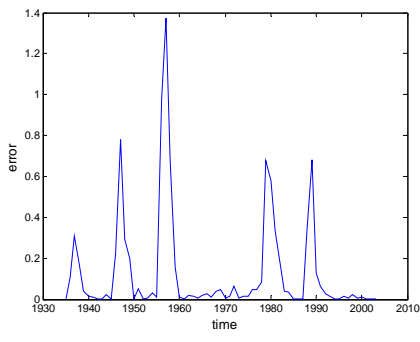

(b) The predicted errors

Fig. 5. Results with iterative-step prediction for sunspot time series by using the Hybrid-II algorithm

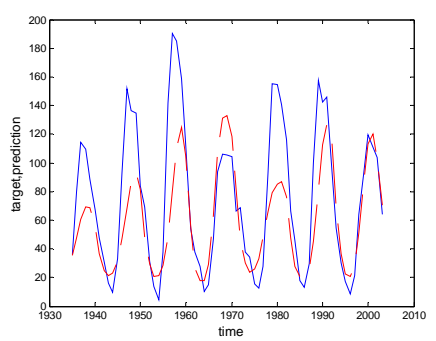

(a) The predicted values

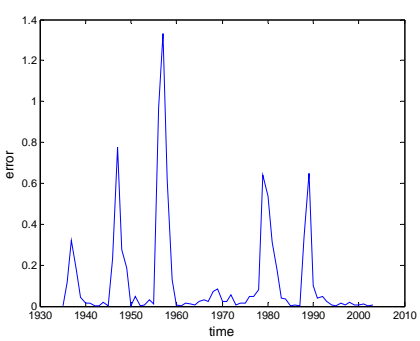

(b) The predicted errors

Fig. 6. Results with iterative-step prediction for sunspot time series by using the new learning algorithm

Table 1. The average values of mean squared errors of single-step prediction for the sunspot time series data for fifty times by four algorithms

\begin{tabular}{clllll}
\hline LA & Training & Testing & Testing & Testing & Testing \\
\hline & $1700-1920$ & $1921-1955$ & $1956-1979$ & $1980-2003$ & $1921-2003$ \\
\hline BP & 0.00090 & 0.00053 & 0.0095 & 0.0090 & 0.0055 \\
\hline Hybrid-I & 0.0021 & 0.00037 & 0.0056 & 0.0040 & 0.0045 \\
\hline Hybrid-II & 0.0021 & 0.00046 & 0.0050 & 0.0044 & 0.0042 \\
\hline New LA & 0.00074 & 0.00030 & 0.0145 & 0.0129 & 0.0031 \\
\hline
\end{tabular}

Table 2. The average values of mean squared errors of iterative-step prediction for the sunspot time series data for fifty times by four algorithms

\begin{tabular}{lll}
\hline LA & Training(1700-1920) & Testing(1921-2003) \\
\hline BP & 0.0391 & 0.1881 \\
\hline Hybrid-I & 0.0390 & 0.1380 \\
\hline Hybrid-II & 0.0401 & 0.1330 \\
\hline New LA & 0.0385 & 0.1285 \\
\hline
\end{tabular}


Below, the effects of the four parameters, $\eta_{1}, \eta_{2}, \gamma_{1}$, and $\gamma_{2}$, with the new modified hybrid learning algorithm for single-step prediction performance on sunspot time series is discussed. Case I: $\eta_{1}=0.3, \eta_{2}=0.15$ and $\gamma_{1}=0.001$ are kept unchanged, $\gamma_{2}$ is selected as $0.001,0.003,0.005$ and 0.007 , respectively. From the simulation results, it can be seen that the bigger the $\gamma_{2}$ is, the worse the generalization performance is. Case II: $\eta_{1}=0.3, \eta_{2}=0.15$ and $\gamma_{2}=0.001$ are kept unchanged, $\gamma_{1}$ is selected as $0.001,0.003,0.005$ and 0.007 , respectively. From the simulation results, it can be seen that the bigger the $\gamma_{1}$ is, the worse the generalization performance is. Case III: $\eta_{1}=0.3, \gamma_{1}=0.001$ and $\gamma_{2}=0.001$ are kept unchanged, $\eta_{2}$ is selected as 0.15 , $0.17,0.19$ and 0.21 , respectively. From the simulation results, it can be seen that the bigger the $\eta_{2}$ is, the worse the generalization performance is. Case IV: $\eta_{2}=0.15$, $\gamma_{1}=0.001$ and $\gamma_{2}=0.001$ are kept unchanged, $\eta_{1}$ is selected as $0.30,0.32,0.34$ and 0.36 , respectively. From the simulation results, it can be seen that the bigger the $\eta_{1}$ is, the worse the generalization performance is. All the above results are shown in Table 3.

Table 3. The effects of the parameters with the new modified hybrid learning algorithm for single-step prediction performance on sunspot time series data

\begin{tabular}{ccccc}
\hline Indices & \multicolumn{4}{c}{ Mean squared errors (1921-2003) } \\
\hline$\eta_{1}=0.3, \eta_{2}=0.15$ & $\gamma_{2}=0.001$ & $\gamma_{2}=0.003$ & $\gamma_{2}=0.005$ & $\gamma_{2}=0.007$ \\
\cline { 2 - 5 }$\gamma_{1}=0.001$ & 0.0031 & 0.0041 & 0.0046 & 0.0050 \\
\hline$\eta_{1}=0.3, \eta_{2}=0.15$ & $\gamma_{1}=0.001$ & $\gamma_{1}=0.003$ & $\gamma_{1}=0.005$ & $\gamma_{1}=0.007$ \\
\cline { 2 - 5 }$\gamma_{2}=0.001$ & 0.0031 & 0.0039 & 0.0042 & 0.0049 \\
\hline$\eta_{1}=0.3, \gamma_{1}=0.001$ & $\eta_{2}=0.15$ & $\eta_{2}=0.17$ & $\eta_{2}=0.19$ & $\eta_{2}=0.21$ \\
\cline { 2 - 5 }$\gamma_{2}=0.001$ & 0.0031 & 0.0038 & 0.0043 & 0.0045 \\
\hline$\eta_{2}=0.15, \gamma_{1}=0.001$ & $\eta_{1}=0.3$ & $\eta_{1}=0.32$ & $\eta_{1}=0.34$ & $\eta_{1}=0.36$ \\
\cline { 2 - 5 }$\gamma_{2}=0.001$ & 0.0031 & 0.0036 & 0.0042 & 0.0046 \\
\hline
\end{tabular}

\subsection{Single-step Prediction for Chaotic Laser Pulsation Data}

In this subsection, the proposed learning algorithm are also applied to the singlestep prediction of chaotic laser pulsation data from the Santa Fe competition data set A. Likely, a (12-8-1)-sized network is also adopted to address this problem. The first 1000 step data are used for the training, and the following 200 steps are used for the single-step prediction test. The predicted results obtained are shown in Fig. 7 for the new learning algorithm.

Similarly, in order to statistically compare the prediction accuracies for chaotic laser pulsation data with the four algorithms (listed in Table4), experiment is also done fifty times for each algorithm and then calculates its average accuracy value. The corresponding results are summarized in Table 4. From these results, it can be 
A Modified Learning Algorithm Incorporating Additional Functional Constraints Into Neural

Networks 9

seen apparently that the proposed learning algorithm has better generalization capability than the BP algorithm as well as the two original hybrid algorithms, since the mean squared errors for the modified learning algorithms for the testing data set are smaller than the ones for the other learning ones.

Obviously, from the above experiments, it can be drawn the conclusion that the new learning algorithm has better generalization performance than the original Hybrid-I and Hybrid-II learning algorithms as well as BP learning algorithm. This result mainly rests with the fact that the new learning one incorporates the additional functional constraints such as the input-to-output mapping sensitivity and the low frequency components in training data into the sum-of-square error cost function.

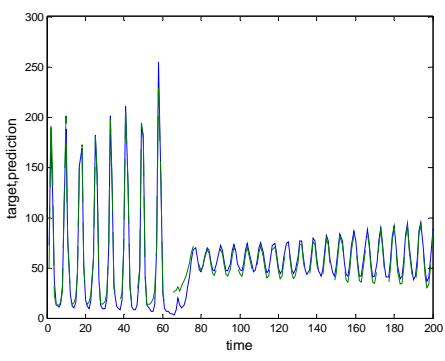

(a) The predicted values

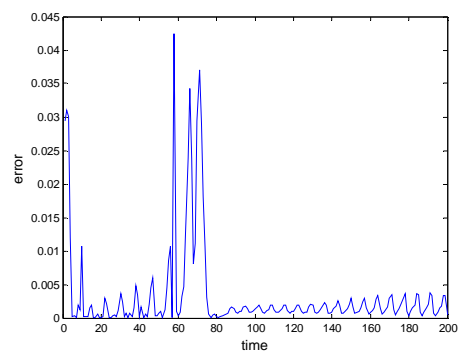

(b) The predicted errors

Fig. 7. Results with single-step prediction for chaotic laser pulsation data by using the new learning algorithm

Table 4. The average values of mean squared errors of single-step prediction for the chaotic laser pulsation data for fifty times by four algorithms

\begin{tabular}{lll}
\hline Learning algorithm & Training & Testing \\
\hline BP & 0.00029725 & 0.0059 \\
\hline Hybrid-I algorithm & 0.0016 & 0.0040 \\
\hline Hybrid-II algorithm & 0.0012 & 0.0043 \\
\hline New LA & 0.00054626 & 0.0033 \\
\hline
\end{tabular}

\section{Conclusions}

In this paper, a new modified learning algorithm with respect to the Hybrid-I and Hybrid-II learning algorithms introduced in literature [10] is proposed. The additional cost terms for this new algorithm are combined with the ones for the Hybrid-I and Hybrid-II learning algorithms and penalize both the input-to-output mapping sensitivity and high frequency components in training data in the course of training, thus the better generalization capability with respect to the original hybrid algorithms can be easily obtained. The experimental results about benchmark data of sunspot time series prediction and chaotic laser pulsation data prediction also showed that the generalization performance of the proposed constrained learning 
algorithm apparently outperforms the one of the Hybrid-I and Hybrid-II learning ones. In addition, the effects of the parameters with the proposed learning algorithm on the network performance were discussed. Future research works will include how to apply this new constrained learning algorithm to resolve more numerical computation problems.

\section{References}

1. D. A. Karras, An efficient constrained training algorithm for feedforward networks, IEEE Trans. Neural Networks 6 (1995) 1420-1434.

2. M. Cottrell, B. Girard et al., Neural modeling for time series: a statistical stepwise method for weight elimination, IEEE Trans. Neural Networks 6 (1992)1355-1364.

3. D.S.Huang, Systematic Theory of Neural Networks for Pattern Recognition. Publishing House of Electronic Industry of China, Beijing, 1996,111-118

4. S.J. Nowlan, G.E. Hinton, Simplifying neural networks by soft weight sharing, Neural Comput. 4 (1992) 473-493.

5. A.S. Weigend, D.E. Rumelhart, B.A. Hyberman, Generalization by weight-elimination applied to currency exchange rate prediction, Proceedings of International Conference on Neural Network, Seattle, 1991, pp.837-841.

6. D.S. Huang, Horace H.S. Ip, Zheru Chi, A Neural Root Finder of Polynomials Based on Root Moments, Neural Computation, 16 (2004) 1721-1762.

7. D.S.Huang, Horace H.S.Ip, Zheru Chi and H.S.Wong, Dilation Method for Finding Close Roots of Polynomials Based on Constrained Learning Neural Networks, Physics Letters A, 309 (2003) 443-451.

8. D.S. Huang, Zheru Chi, Finding roots of arbitrary high order polynomials based on neural network recursive partitioning method, Science in China Ser. F Information Sciences, 47 (2004) 232-245.

9. D. S. Huang, A constructive approach for finding arbitrary roots of polynomials by neural networks, IEEE Transactions on Neural Networks, 15 (2004) 477-491.

10. S.Y. Jeong, S.Y. Lee, Adaptive learning algorithms to incorporate additional functional constraints into neural networks, Neurocomputing, 35 (2000), 73-90.

11. D.-G. Jeong, S.-Y. Lee, Merging back-propagation and Hebbian learning rules for robust classifications, Neural Networks 9 (1996) 1213-1222.

12. C. Goutte, L.K. Hansen, Regularization with a pruning prior, Neural Networks 10 (1997) 1053-1059.

13. P.M. Williams, Bayesian regularization and pruning using a Laplace prior, Neural Comput. 7 (1995) 117-143. 